\title{
Trigonometric approximation of functions $f(x, y)$ of generalized Lipschitz class by double Hausdorff matrix summability method
}

\author{
Abhishek Mishra ${ }^{1}$, Vishnu Narayan Mishra ${ }^{2}$ and M. Mursaleen ${ }^{3,4^{*}}$ (I)
}

\author{
"Correspondence: \\ mursaleenm@gmail.com \\ ${ }^{3}$ Department of Medical Research, \\ China Medical University Hospital, \\ China Medical University (Taiwan), \\ Taichung, Taiwan \\ ${ }^{4}$ Department of Mathematics, \\ Aligarh Muslim University, Aligarh, \\ India \\ Full list of author information is \\ available at the end of the article
}

\begin{abstract}
In this paper, we establish a new estimate for the degree of approximation of functions $f(x, y)$ belonging to the generalized Lipschitz class $L i p\left(\left(\xi_{1}, \xi_{2}\right) ; r\right), r \geq 1$, by double Hausdorff matrix summability means of double Fourier series. We also deduce the degree of approximation of functions from $\operatorname{Lip}((\alpha, \beta) ; r)$ and $\operatorname{Lip}(\alpha, \beta)$ in the form of corollary. We establish some auxiliary results on trigonometric approximation for almost Euler means and $(C, \gamma, \delta)$ means.
\end{abstract}

Keywords: Double Hausdorff matrix summability; Double Fourier series; Generalized Lipschitz class; Modulus of continuity; Cesàro summability; Almost Euler summability means; Degree of approximation

\section{Introduction}

The study of various summability means of double Fourier series have been done by several authors, for example, Chow [2], Sharma [11], Łenski [6], and Ustina [15]. Dealing with the first arithmetic means of double Fourier series, Hasegawa [4] obtained the following:

Theorem A If a continuous function $f(x, y)$ of period $2 \pi$ with respect to both $x$ and $y$ belongs to $\operatorname{Lip}(\alpha, \beta)$, where $0<\alpha<$ land $0<\beta<1$, then

$$
\left|\sigma_{m, n}(x, y)-f(x, y)\right|=O\left(m^{-\alpha}+n^{-\beta}\right)
$$

uniformly in $(x, y)$ as $m$ and $n$ independently tend to infinity.

If $\alpha=\beta=1$, then

$$
\left|\sigma_{m, n}(x, y)-f(x, y)\right|=O\left(m^{-1} \log m+n^{-1} \log n\right)
$$

uniformly in $(x, y)$ as $m$ and $n$ independently tend to infinity.

Siddiqui and Mohammadzadeh [12] investigated the approximation by Cesàro and $\mathrm{B}$ means of double Fourier series. Stepanets $[13,14]$ has established estimates of approximation for certain classes of periodic functions and differentiable periodic functions of two

(c) The Author(s) 2020. This article is licensed under a Creative Commons Attribution 4.0 International License, which permits use, sharing, adaptation, distribution and reproduction in any medium or format, as long as you give appropriate credit to the original author(s) and the source, provide a link to the Creative Commons licence, and indicate if changes were made. The images or other third party material in this article are included in the article's Creative Commons licence, unless indicated otherwise in a credit line to the material. If material is not included in the article's Creative Commons licence and your intended use is not permitted by statutory regulation or exceeds the permitted use, you will need to obtain permission directly from the copyright holder. To view a copy of this licence, visit http://creativecommons.org/licenses/by/4.0/ 
variables by linear methods of summation of their Fourier sums. Móricz and Shi [8] proved the following result for the approximation to continuous functions by Cesàro means of double Fourier series.

Theorem B If $f \in E(\alpha, \beta), 0<\alpha, \beta \leq 1, \gamma, \delta \geq 0$, then

$$
\begin{aligned}
\| \sigma_{m n}^{\gamma \delta}(f, x, y)-f(x, y \| & =O\left(\frac{1}{(m+1)^{\alpha}}+\frac{1}{(n+1)^{\beta}}\right) \quad \text { if } 0<\alpha, \beta \leq 1, \\
& =O\left(\frac{1}{(m+1)^{\alpha}}+\frac{\log (n+2)}{(n+1)}\right) \quad \text { if } 0<\alpha<\beta=1, \\
& =O\left(\frac{\log (m+2)}{(m+1)}+\frac{\log (n+2)}{(n+1)}\right) \quad \text { if } \alpha=\beta=1 .
\end{aligned}
$$

The degree of approximation using Gauss-Weierstrass integrals was also investigated by Khan and Ram [5]. Recently, error and bounds of certain bivariate functions by almost Euler means of double Fourier series for the functions of Lipschitz and Zygmund classes was estimated by Rathor and Singh [9]. To find the approximation of functions of two-dimensional torus, in this paper, we obtain a new estimate for trigonometric approximation of functions $f(x, y)$ of generalized Lipschitz class by double Hausdorff matrix summability method of double Fourier series. For other summability methods of approximation, see [1] and [7].

\section{Definitions and preliminaries}

Let $\sum_{m=0}^{\infty} \sum_{n=0}^{\infty} g_{m, n}$ be double series with the sequence of $(m, n)$ th partial sums

$$
s_{m, n}=\sum_{j=0}^{m} \sum_{k=0}^{n} g_{j, k} \text {. }
$$

A double Hausdorff matrix has the entries

$$
h_{m, n}^{j, k}=\left(\begin{array}{c}
m \\
j
\end{array}\right)\left(\begin{array}{l}
n \\
k
\end{array}\right) \Delta_{1}^{m-j} \Delta_{2}^{n-k} \mu_{j, k}
$$

where $\left\{\mu_{j, k}\right\}$ is any real or complex sequence, and

$$
\Delta_{1}^{m-j} \Delta_{2}^{n-k} \mu_{j, k}=\sum_{w=0}^{m-j} \sum_{z=0}^{n-k}(-1)^{j+k}\left(\begin{array}{c}
m-j \\
w
\end{array}\right)\left(\begin{array}{c}
n-k \\
z
\end{array}\right) \mu_{j+w, k+z} .
$$

If $t_{m, n}^{H}=\sum_{j=0}^{m} \sum_{k=0}^{n} h_{m, n}^{j, k} s_{j, k} \rightarrow g$ as $m \rightarrow \infty$ and $n \rightarrow \infty$, then $\sum_{m=0}^{\infty} \sum_{n=0}^{\infty} g_{m, n}$ is said to be summable to the sum $g$ by the double Hausdorff matrix summability method [15].

A necessary and sufficient condition for double Hausdorff matrix summability method to be regular is there exists a function $\chi(s, t) \in B V[0,1] \times[0,1]$ such that

$$
\int_{0}^{1} \int_{0}^{1}|d \chi(s, t)|<\infty
$$

and

$$
\mu_{m, n}=\int_{0}^{1} \int_{0}^{1} s^{m} t^{n} d \chi(s, t),
$$


where $\chi(s, 0)=\chi\left(s, 0^{+}\right)=\chi\left(0^{+}, t\right)=\chi(0, t)=0,0 \leq s, t \leq 1$, and $\chi(1,1)-\chi(1,0)-\chi(0,1)+$ $\chi(0,0)=1[10]$.

It is easy to see that the absolute value of the measure $d \chi(s, t)$ can me majorized by $K_{1} K_{2} d s d t$ for some constants $K_{1}$ and $K_{2}$ (see [16]).

The important particular cases of double Hausdorff matrix summability means are as follows:

1 Almost Euler summability means $\left(\left(E, q_{1}, q_{2}\right)\right.$ means $)$ if $\mu_{m, n}=\frac{1}{\left(1+q_{1}\right)^{m}} \frac{1}{\left(1+q_{2}\right)^{n}}$.

$2(E, 1,1)$ means if $q_{1}=1$ and $q_{2}=1$ in $\left(E, q_{1}, q_{2}\right)$ means.

$3(C, \gamma, \delta)$ means if $\mu_{m, n}=\frac{1}{A_{m}^{\gamma}} \frac{1}{A_{n}^{\delta}}$, where $\gamma, \delta \geq-1$ and $A_{m}^{\gamma}=\left(\begin{array}{c}\gamma+m \\ m\end{array}\right), A_{n}^{\delta}=\left(\begin{array}{c}\delta+n \\ n\end{array}\right)$.

$4(C, 1,1)$ means if $\gamma=\delta=1$ in $(C, \gamma, \delta)$ means.

Let $f(x, y)$ be a Lebesgue-integrable function of period $2 \pi$ with respect to both variables $x$ and $y$ and summable in the fundamental square $Q:(-\pi, \pi) \times(-\pi, \pi)$. The double Fourier series of $f(x, y)$ is given by

$$
\begin{aligned}
f(x, y)= & \sum_{m=0}^{\infty} \sum_{n=0}^{\infty} \lambda_{m, n}\left[a_{m, n} \cos m x \cos n y+b_{m, n} \sin m x \cos n y\right. \\
& \left.+c_{m, n} \cos m x \sin n y+d_{m, n} \sin m x \cos n y\right]
\end{aligned}
$$

with $(m, n)$ th partial sums $s_{m, n}(f ;(x, y))$, where

$$
\begin{aligned}
& \lambda_{m, n}= \begin{cases}1 / 4 & \text { for } m=n=0, \\
1 / 2 & \text { for } m>0, n=0 \text { and } m=0, n>0, \\
1 & \text { for } m>0, n>0,\end{cases} \\
& a_{m, n}=\pi^{-2} \iint_{Q} f(x, y) \cos m x \cos n y d x d y,
\end{aligned}
$$

and similar expressions for $b_{m, n}, c_{m, n}$, and $d_{m, n}$ [3].

We define the $L^{r}$ norm by

$$
\|f\|_{r}= \begin{cases}\left\{\frac{1}{4 \pi} \int_{0}^{2 \pi} \int_{0}^{2 \pi}|f(x, y)|^{r} d x d y\right\}^{1 / r}, & r \geq 1, \\ \operatorname{ess~sup}_{0 \leq x, y \leq 2 \pi}|f(x, y)|, & r=\infty\end{cases}
$$

The degree of approximation of a function $f: \mathbb{R}^{2} \rightarrow \mathbb{R}$ by a trigonometric polynomial [17]

$$
\begin{aligned}
t_{m, n}(x, y)= & \sum_{j=0}^{m} \sum_{k=0}^{n} \lambda_{m, n}\left[a_{j, k} \cos m x \cos n y+b_{j, k} \sin m x \cos n y\right. \\
& \left.+c_{j, k} \cos m x \sin n y+d_{j, k} \sin m x \cos n y\right]
\end{aligned}
$$

of order $(m+n)$ is defined by

$$
E_{m, n}\left(f, L^{r}\right)=\min _{0 \leq x, y \leq 2 \pi}\left\|t_{m, n}-f\right\|_{r}
$$


A function $f: \mathbb{R}^{2} \rightarrow \mathbb{R}$ of two variables $x$ and $y$ is said to belong to the class $\operatorname{Lip}(\alpha, \beta)[4]$ if

$$
|f(x+u, y+v)-f(x, y)|=O\left(|u|^{\alpha}+|v|^{\beta}\right), \quad 0<\alpha \leq 1,0<\beta \leq 1,
$$

to the class $\operatorname{Lip}((\alpha, \beta) ; r)$ if

$$
\left\{\frac{1}{4 \pi} \int_{0}^{2 \pi} \int_{0}^{2 \pi}|f(x+u, y+v)-f(x, y)|^{r} d x d y\right\}^{1 / r}=O\left(|u|^{\alpha}+|v|^{\beta}\right), \quad r \geq 1,
$$

and to the class $\operatorname{Lip}\left(\left(\xi_{1}, \xi_{2}\right) ; r\right)$ if

$$
\left\{\frac{1}{4 \pi} \int_{0}^{2 \pi} \int_{0}^{2 \pi}|f(x+u, y+v)-f(x, y)|^{r} d x d y\right\}^{1 / r}=O\left(\xi_{1}(u)+\xi_{2}(v)\right), \quad r \geq 1,
$$

where $\xi_{1}$ and $\xi_{2}$ are moduli of continuity, that is, nonnegative nondecreasing continuous functions such that $\xi_{1}(0)=\xi_{2}(0)=0, \xi_{1}\left(u_{1}+u_{2}\right) \leq \xi_{1}\left(u_{1}\right)+\xi_{1}\left(u_{2}\right)$, and $\xi_{2}\left(v_{1}+v_{2}\right) \leq \xi_{2}\left(v_{1}\right)+$ $\xi_{2}\left(v_{2}\right)$.

If $\xi_{1}(u)=u^{\alpha}$ and $\xi_{2}(v)=v^{\beta}, 0<\alpha \leq 1,0<\beta \leq 1$, then the class $\operatorname{Lip}\left(\left(\xi_{1}, \xi_{2}\right) ; r\right)$ coincides with $\operatorname{Lip}((\alpha, \beta) ; r)$. As $r \rightarrow \infty, \operatorname{Lip}((\alpha, \beta) ; r)$ reduces to $\operatorname{Lip}(\alpha, \beta)$. Clearly, $\operatorname{Lip}(\alpha, \beta) \subseteq$ $\operatorname{Lip}((\alpha, \beta) ; r) \subseteq \operatorname{Lip}\left(\left(\xi_{1}, \xi_{2}\right) ; r\right)$.

We define the forward difference operator $\Delta$ as $\Delta \mu_{k}=\mu_{k}-\mu_{k+1}$; also, $\Delta^{n+1} \mu_{k}=$ $\Delta\left(\Delta^{n} \mu_{k}\right), k \geq 0$. We denote

$$
\begin{aligned}
\phi(u, v)= & (1 / 4)[f(x+u, y+v)+f(x+u, y-v)+f(x-u, y+v)+f(x-u, y-v) \\
& -4 f(x, y)], \\
M_{m}^{H}(u)= & \frac{K_{1}}{2 \pi} \sum_{j=0}^{m} \int_{0}^{1}\left(\begin{array}{c}
m \\
j
\end{array}\right) s^{j}(1-s)^{m-j} d s \frac{\sin \left(j+\frac{1}{2}\right) u}{\sin \frac{u}{2}}, \\
K_{n}^{H}(v)= & \frac{K_{2}}{2 \pi} \sum_{k=0}^{n} \int_{0}^{1}\left(\begin{array}{l}
N \\
K
\end{array}\right) t^{k}(1-t)^{n-k} d t \frac{\sin \left(k+\frac{1}{2}\right) v}{\sin \frac{v}{2}} .
\end{aligned}
$$

\section{Result}

The object of this paper is obtaining the degree of approximation of functions $f(x, y)$ of generalized Lipschitz class by double Hausdorff matrix summability means of its double Fourier series:

Theorem 1 If $f(x, y)$ is a $2 \pi$ periodic function with respect to both variables $x$ and $y$, Lebesgue integrable in $(-\pi, \pi) \times(-\pi, \pi)$ and belonging to the class Lip $\left(\left(\xi_{1}, \xi_{2}\right) ; r\right)(r \geq 1)$, then the degree of approximation of $f(x, y)$ by double Hausdorff matrix summability means

$$
t_{m, n}^{H}=\sum_{j=0}^{m} \sum_{k=0}^{n} \int_{0}^{1} \int_{0}^{1}\left(\begin{array}{c}
m \\
j
\end{array}\right)\left(\begin{array}{l}
n \\
k
\end{array}\right) s^{j}(1-s)^{m-j} t^{k}(1-t)^{n-k} d \chi(s, t) s_{j, k}
$$


of double Fourier series (1) satisfies

$$
\begin{aligned}
& \left\|t_{m, n}^{H}-f\right\|_{r}=O\left(\frac{1}{(m+1)} \int_{\frac{1}{m+1}}^{\pi} \frac{\xi_{1}(u)}{u^{2}} d u+\frac{1}{(n+1)} \int_{\frac{1}{n+1}}^{\pi} \frac{\xi_{2}(v)}{v^{2}} d v\right) \\
& \quad \text { for } m, n=0,1,2, \ldots
\end{aligned}
$$

\section{Lemmas}

For the proof of our theorems, we need the following lemmas.

Lemma $1\left|M_{m}^{H}(u)\right|=O(m+1)$ for $0<u \leq \frac{1}{m+1}$, and $\left|K_{n}^{H}(v)\right|=O(n+1)$ for $0<v \leq \frac{1}{n+1}$.

Proof Since $|\sin m u| \leq m u$ for $0<u \leq \frac{1}{m+1}$ and $\sin (u / 2) \geq(u / \pi)$, we have

$$
\begin{aligned}
\left|M_{m}^{H}(u)\right| & =\left|\frac{K_{1}}{2 \pi} \sum_{j=0}^{m} \int_{0}^{1}\left(\begin{array}{c}
m \\
j
\end{array}\right) s^{j}(1-s)^{m-j} d s \frac{\sin \left(j+\frac{1}{2}\right) u}{\sin \frac{u}{2}}\right| \\
& =\frac{K_{1}}{2 \pi} \sum_{j=0}^{m} \int_{0}^{1}\left(\begin{array}{c}
m \\
j
\end{array}\right) s^{j}(1-s)^{m-j} d s \frac{\left|\sin \left(j+\frac{1}{2}\right) u\right|}{\left|\sin \frac{u}{2}\right|} \\
& \leq \frac{K_{1}}{2 \pi} \sum_{j=0}^{m} \int_{0}^{1}\left(\begin{array}{c}
m \\
j
\end{array}\right) s^{j}(1-s)^{m-j} d s \frac{\left(j+\frac{1}{2}\right) u}{\left|\frac{u}{\pi}\right|} \\
& =K_{1} \pi\left(m+\frac{1}{2}\right) \int_{0}^{1} \sum_{j=0}^{m}\left(\begin{array}{c}
m \\
j
\end{array}\right) s^{j}(1-s)^{m-j} d s \\
& =K_{1} \pi\left(m+\frac{1}{2}\right) \int_{0}^{1}(s+1-s)^{m} d s \\
& =O(m+1) .
\end{aligned}
$$

Similarly, for $0<v \leq \frac{1}{n+1}$,

$$
\left|K_{n}^{H}(v)\right|=O(n+1) .
$$

Lemma $2\left|M_{m}^{H}(u)\right|=O\left(\frac{1}{(j+1) u^{2}}\right)$ for $\frac{1}{m+1}<u \leq \pi$, and $\left|K_{n}^{H}(v)\right|=O\left(\frac{1}{(k+1) v^{2}}\right)$ for $\frac{1}{n+1}<v \leq \pi$.

Proof Since $\sin (m+1) u \leq 1$ for $\frac{1}{m+1}<u \leq \pi$ and $\sin (u / 2) \geq(u / \pi)$, we get

$$
\begin{aligned}
\left|\sum_{j=0}^{m} \int_{0}^{1}\left(\begin{array}{c}
m \\
j
\end{array}\right) s^{j}(1-s)^{m-j} e^{i\left(j+\frac{1}{2}\right) u} d s\right| & =\int_{0}^{1} e^{i u / 2} \sum_{j=0}^{m}\left(\begin{array}{c}
m \\
j
\end{array}\right) s^{j}(1-s)^{m-j} e^{i j u} d s \\
& =\int_{0}^{1} e^{i u / 2}\left(1-s+s e^{i u}\right)^{m} d s \\
& =O\left(\frac{1}{(m+1)}\right)\left(\frac{e^{i u / 2}\left(e^{i(m+1) u}-1\right)}{e^{i u}-1}\right) .
\end{aligned}
$$

Equating the imaginary parts of both sides, we get

$$
\left|\sum_{j=0}^{m} \int_{0}^{1}\left(\begin{array}{c}
m \\
j
\end{array}\right) s^{k}(1-s)^{m-j} \sin \left(k+\frac{1}{2}\right) d s\right|=O\left(\frac{1}{(m+1) u}\right) .
$$


Therefore

$$
\begin{aligned}
\left|M_{m}^{H}(u)\right| & =\left|\frac{K_{1}}{2 \pi} \sum_{j=0}^{m} \int_{0}^{1}\left(\begin{array}{c}
m \\
j
\end{array}\right) s^{j}(1-s)^{m-j} \frac{\sin \left(j+\frac{1}{2}\right) u}{\sin \frac{u}{2}} d s\right| \\
& \leq \frac{K_{1}}{2 u}\left|\sum_{j=0}^{m} \int_{0}^{1}\left(\begin{array}{c}
m \\
j
\end{array}\right) s^{j}(1-s)^{m-j} \sin \left(j+\frac{1}{2}\right) u d s\right| \\
& =O\left(\frac{1}{(m+1) u^{2}}\right) .
\end{aligned}
$$

Similarly, for $\frac{1}{n+1}<v \leq \pi$,

$$
\left|K_{n}^{H}(v)\right|=O\left(\frac{1}{(n+1) v^{2}}\right) .
$$

Lemma 3 If $f(x, y) \in \operatorname{Lip}\left(\left(\xi_{1}, \xi_{2}\right) ; r\right)(r \geq 1)$, then $\left.\| \phi(u, v)\right) \|_{r}=O\left(\xi_{1}(u)+\xi_{2}(v)\right)$.

Proof Clearly,

$$
\begin{aligned}
|\phi(u, v)|= & \frac{1}{4}|f(x+u, y+v)+f(x+u, y-v)+f(x-u, y+v)+f(x-u, y-v)-4 f(x, y)| \\
\leq & \frac{1}{4}[|f(x+u, y+v)-f(x, y)|+|f(x+u, y-v)-f(x, y)| \\
& +|f(x-u, y+v)-f(x, y)|+|f(x-u, y-v)-f(x, y)|] \\
\|\phi(u, v)\|_{r} \leq & \frac{1}{4}\left[\|f(x+u, y+v)-f(x, y)\|_{r}+\|f(x+u, y-v)-f(x, y)\|_{r}\right. \\
& \left.+\|f(x-u, y+v)-f(x, y)\|_{r}+\|f(x-u, y-v)-f(x, y)\|_{r}\right] \\
= & O\left(\xi_{1}(u)+\xi_{2}(v)\right) .
\end{aligned}
$$

\section{Proof of Theorem 1}

The $(m, n)$ th partial sum of the double Fourier series (1) is given by

$$
s_{m, n}(f ;(x, y))-f(x, y)=\frac{1}{4 \pi^{2}} \int_{0}^{\pi} \int_{0}^{\pi} \phi(u, v) \frac{\sin \left(m+\frac{1}{2}\right) u \sin \left(n+\frac{1}{2}\right) v}{\sin \frac{u}{2} \sin \frac{v}{2}} d u d v .
$$

Denoting the double Hausdorff matrix sums of $s_{m, n}$ by $t_{m, n}^{H}$, we have

$$
\begin{aligned}
t_{m, n}^{H}(x, y)-f(x, y) & =\sum_{j=0}^{m} \sum_{k=0}^{n} h_{m, n}^{j, k}\left\{s_{j, k}(f ;(x, y))-f(x, y)\right\} \\
& =\int_{0}^{\pi} \int_{0}^{\pi} \phi(u, v) \sum_{j=0}^{m} \sum_{k=0}^{n} h_{m, n}^{j, k} \frac{\sin \left(j+\frac{1}{2}\right) u \sin \left(k+\frac{1}{2}\right) v}{\sin \frac{u}{2} \sin \frac{v}{2}} d u d v \\
& =\int_{0}^{\pi} \int_{0}^{\pi} \phi(u, v) M_{m}^{H}(u) K_{n}^{H}(v) d u d v,
\end{aligned}
$$


Mishra et al. Advances in Difference Equations

(2020) 2020:681

Page 7 of 10

$$
\begin{aligned}
\left\|t_{m, n}^{H}-f\right\|_{r}= & \int_{0}^{\pi} \int_{0}^{\pi}\|\phi(u, v)\|_{r} M_{m}^{H}(u) K_{n}^{H}(v) d u d v \\
= & \left(\int_{0}^{\frac{1}{m+1}} \int_{0}^{\frac{1}{n+1}}+\int_{0}^{\frac{1}{m+1}} \int_{\frac{1}{n+1}}^{\pi}+\int_{\frac{1}{m+1}}^{\pi} \int_{0}^{\frac{1}{n+1}}+\int_{\frac{1}{m+1}}^{\pi} \int_{\frac{1}{n+1}}^{\pi}\right) \\
& \|\phi(u, v)\|_{r} M_{m}^{H}(u) K_{n}^{H}(v) d u d v \\
= & I_{1}+I_{2}+I_{3}+I_{4}, \quad \text { say. }
\end{aligned}
$$

Using Lemmas 1 and 3, we obtain

$$
\begin{aligned}
\left|I_{1}\right| & =\int_{0}^{\frac{1}{m+1}} \int_{0}^{\frac{1}{n+1}}\|\phi(u, v)\|_{r} M_{m}^{H}(u) K_{n}^{H}(v) d u d v \\
& =O\left(\int_{0}^{\frac{1}{m+1}} \int_{0}^{\frac{1}{n+1}}\left(\xi_{1}(u)+\xi_{2}(v)\right)(m+1)(n+1) d u d v\right) \\
& =O\left((m+1)(n+1) \int_{0}^{\frac{1}{m+1}} \int_{0}^{\frac{1}{n+1}}\left(\xi_{1}(u)+\xi_{2}(v)\right) d u d v\right) \\
& =O\left[(m+1)(n+1)\left(\int_{0}^{\frac{1}{m+1}} \int_{0}^{\frac{1}{n+1}} \xi_{1}(u) d u d v+\int_{0}^{\frac{1}{m+1}} \int_{0}^{\frac{1}{n+1}} \xi_{2}(v) d u d v\right)\right] \\
& =O\left[(m+1)(n+1)\left(\int_{0}^{\frac{1}{m+1}} \frac{\xi_{1}(u)}{n+1} d u+\int_{0}^{\frac{1}{m+1}} \frac{\xi_{2}\left(\frac{1}{(n+1)}\right)}{n+1} d v\right)\right] \\
& =O\left[(m+1)(n+1)\left(\frac{\xi_{1}\left(\frac{1}{(m+1)}\right)}{(m+1)(n+1)}+\frac{\xi_{2}\left(\frac{1}{(n+1)}\right)}{(m+1)(n+1)}\right)\right] \\
& =O\left(\xi_{1}\left(\frac{1}{m+1}\right)+\xi_{2}\left(\frac{1}{n+1}\right)\right) .
\end{aligned}
$$

Again by Lemmas 1-3, we have

$$
\begin{aligned}
\left|I_{2}\right| & =O\left[\int_{0}^{\frac{1}{m+1}} \int_{\frac{1}{n+1}}^{\pi}\left(\xi_{1}(u)+\xi_{2}(v)\right) \frac{(m+1)}{(n+1) v^{2}} d u d v\right] \\
& =O\left[\frac{(m+1)}{(n+1)}\left(\int_{0}^{\frac{1}{m+1}} \xi_{1}(u) d u \int_{\frac{1}{n+1}}^{\pi} \frac{d v}{v^{2}}+\int_{0}^{\frac{1}{m+1}} d u \int_{\frac{1}{n+1}}^{\pi} \frac{\xi_{2}(v)}{v^{2}} d v\right)\right] \\
& =O\left[\frac{(m+1)}{(n+1)}\left(\xi_{1}\left(\frac{1}{m+1}\right) \frac{1}{(m+1)}\left((n+1)-\frac{1}{\pi}\right)+\frac{1}{(m+1)} \int_{\frac{1}{n+1}}^{\pi} \frac{\xi_{2}(v)}{v^{2}} d v\right)\right] \\
& =O\left(\xi_{1}\left(\frac{1}{m+1}\right)+\frac{1}{(n+1)} \int_{\frac{1}{n+1}}^{\pi} \frac{\xi_{2}(v)}{v^{2}} d v\right) .
\end{aligned}
$$

Similarly,

$$
\begin{aligned}
\left|I_{3}\right| & =O\left[\int_{\frac{1}{m+1}}^{\pi} \int_{0}^{\frac{1}{n+1}}\left(\xi_{1}(u)+\xi_{2}(v)\right) \frac{(n+1)}{(m+1) u^{2}} d u d v\right] \\
& =O\left[\frac{(n+1)}{(m+1)}\left(\int_{\frac{1}{m+1}}^{\pi} \frac{\xi_{1}(u)}{u^{2}} d u \int_{0}^{\frac{1}{n+1}} d v+\int_{\frac{1}{m+1}}^{\pi} \frac{d u}{u^{2}} \int_{0}^{\frac{1}{n+1}} \xi_{2}(v) d v\right)\right] \\
& =O\left(\frac{1}{(m+1)} \int_{\frac{1}{m+1}}^{\pi} \frac{\xi_{1}(u)}{u^{2}} d u+\xi_{2}\left(\frac{1}{n+1}\right)\right) .
\end{aligned}
$$


Also, using Lemmas 2 and 3, we get

$$
\begin{aligned}
\left|I_{4}\right| & =O\left[\int_{\frac{1}{m+1}}^{\pi} \int_{\frac{1}{n+1}}^{\pi}\left(\xi_{1}(u)+\xi_{2}(v)\right) \frac{1}{(m+1) u^{2}} \frac{1}{(n+1) v^{2}} d u d v\right] \\
& =O\left[\frac{1}{(m+1)(n+1)}\left(\int_{\frac{1}{m+1}}^{\pi} \frac{\xi_{1}}{u^{2}} d u \int_{\frac{1}{n+1}}^{\pi} \frac{1}{v^{2}} d v+\int_{\frac{1}{m+1}}^{\pi} \frac{1}{u^{2}} d u \int_{\frac{1}{n+1}}^{\pi} \frac{\xi_{2}}{v^{2}} d v\right)\right] \\
& =O\left(\frac{1}{(m+1)} \int_{\frac{1}{m+1}}^{\pi} \frac{\xi_{1}(u)}{u^{2}} d u+\frac{1}{(n+1)} \int_{\frac{1}{n+1}}^{\pi} \frac{\xi_{2}(v)}{v^{2}} d v\right) .
\end{aligned}
$$

Next,

$$
\begin{aligned}
& \frac{1}{(m+1)} \int_{\frac{1}{m+1}}^{\pi} \frac{\xi_{1}(u)}{u^{2}} d u \geq \frac{1}{(m+1)} \xi_{1}\left(\frac{1}{m+1}\right) \int_{\frac{1}{m+1}}^{\pi} \frac{1}{u^{2}} d t \\
&=\frac{1}{(m+1)} \xi_{1}\left(\frac{1}{m+1}\right)\left\{-\frac{1}{u}\right\}_{\frac{1}{m+1}}^{\pi} \\
&=\xi_{1}\left(\frac{1}{m+1}\right)\left\{1-\frac{1}{(m+1) \pi}\right\} \\
& \geq \frac{1}{2} \xi_{1}\left(\frac{1}{m+1}\right), \\
& \text { or } \xi_{1}\left(\frac{1}{m+1}\right)=O\left(\frac{1}{(m+1)} \int_{\frac{1}{m+1}}^{\pi} \frac{\xi_{1}(u)}{u^{2}} d t\right) .
\end{aligned}
$$

Similarly,

$$
\xi_{2}\left(\frac{1}{(n+1)}\right)=O\left(\frac{1}{(n+1)} \int_{\frac{1}{n+1}}^{\pi} \frac{\xi_{2}(v)}{v^{2}} d t\right)
$$

Combining equations (5)-(10), we have

$$
\left\|t_{m, n}^{H}-f\right\|_{r}=O\left(\frac{1}{(m+1)} \int_{\frac{1}{m+1}}^{\pi} \frac{\xi_{1}(u)}{u^{2}} d u+\frac{1}{(n+1)} \int_{\frac{1}{n+1}}^{\pi} \frac{\xi_{2}(v)}{v^{2}} d v\right) .
$$

This completes the proof of Theorem 1 .

\section{Corollaries}

From the main theorem we derive the following corollaries.

Corollary 1 If $f(x, y)$ is a $2 \pi$ periodic function with respect to both variables $x$ and $y$, Lebesgue integrable in $(-\pi, \pi) \times(-\pi, \pi)$ and belonging to the class Lip $((\alpha, \beta) ; r)(r \geq 1)$, then the degree of approximation off $(x, y)$ by means $t_{m, n}^{H}$ of double Fourier series (1) satisfies

$$
\left\|t_{m, n}^{H}-f\right\|_{r}= \begin{cases}O\left((m+1)^{-\alpha}+(n+1)^{-\beta}\right), & 0<\alpha<1,0<\beta<1, \\ O\left((m+1)^{-\alpha}+\frac{\log (n+1) \pi}{(n+1)}\right), & 0<\alpha<1, \beta=1, \\ O\left(\frac{\log (m+1) \pi}{(m+1)}+(n+1)^{-\beta}\right), & \alpha=1,0<\beta<1, \\ O\left(\frac{\log (m+1) \pi}{(m+1)}+\frac{\log (n+1) \pi}{(n+1)}\right), & \alpha=\beta=1,\end{cases}
$$

for $m, n=0,1,2, \ldots$ 
Corollary 2 If $f(x, y)$ is a $2 \pi$ periodic function with respect to both variables $x$ and $y$, Lebesgue integrable in $(-\pi, \pi) \times(-\pi, \pi)$ and belonging to the class Lip $(\alpha, \beta)$, then the degree of approximation of $f(x, y)$ by double Hausdorff matrix summability means $t_{m, n}^{H}$ of double Fourier series (1) satisfies

$$
\left\|t_{m, n}^{H}-f\right\|_{\infty}= \begin{cases}O\left((m+1)^{-\alpha}+(n+1)^{-\beta}\right), & 0<\alpha<1,0<\beta<1, \\ O\left((m+1)^{-\alpha}+\frac{\log (n+1) \pi}{(n+1)}\right), & 0<\alpha<1, \beta=1, \\ O\left(\frac{\log (m+1) \pi}{(m+1)}+(n+1)^{-\beta}\right), & \alpha=1,0<\beta<1, \\ O\left(\frac{\log (m+1) \pi}{(m+1)}+\frac{\log (n+1) \pi}{(n+1)}\right), & \alpha=\beta=1,\end{cases}
$$

for $m, n=0,1,2, \ldots$

Corollary 3 If $f(x, y)$ is a $2 \pi$ periodic function with respect to both variables $x$ and $y$, Lebesgue integrable in $(-\pi, \pi) \times(-\pi, \pi)$ and belonging to the class $\operatorname{Lip}\left(\left(\xi_{1}, \xi_{2}\right) ; r\right)$, then the degree of approximation of $f(x, y)$ by almost Euler summability means

$$
t_{m, n}^{E}=\frac{1}{\left(1+q_{1}\right)^{m}} \frac{1}{\left(1+q_{2}\right)^{n}} \sum_{j=0}^{m} \sum_{k=0}^{n}\left(\begin{array}{c}
m \\
j
\end{array}\right)\left(\begin{array}{l}
n \\
k
\end{array}\right) q_{1}^{m-j} q_{2}^{n-k} s_{j, k}
$$

of double Fourier series (1) satisfies

$$
\left\|t_{m, n}^{E}-f\right\|_{r}=O\left(\frac{1}{(m+1)} \int_{\frac{1}{m+1}}^{\pi} \frac{\xi_{1}(u)}{u^{2}} d u+\frac{1}{(n+1)} \int_{\frac{1}{n+1}}^{\pi} \frac{\xi_{2}(v)}{v^{2}} d v\right)
$$

for $m, n=0,1,2, \ldots$

Corollary 4 For $\gamma, \delta \geq-1$, the Cesàro means $\sigma_{m, n}^{\gamma, \delta}$ of order $\gamma$ and $\delta$, that is, $(C, \gamma, \delta)$ means of double Fourier series, are given by

$$
\sigma_{m, n}^{\gamma, \delta}=\frac{1}{A_{m}^{\gamma}} \frac{1}{A_{n}^{\delta}} \sum_{j=0}^{m} \sum_{k=0}^{n} A_{m-j}^{\gamma-1} A_{n-k}^{\delta-1} s_{j, k},
$$

where $A_{m}^{\gamma}=\left(\begin{array}{c}\gamma+m \\ m\end{array}\right)$ and $A_{n}^{\delta}=\left(\begin{array}{c}\delta+n \\ n\end{array}\right)$.

If $f(x, y)$ is a $2 \pi$ periodic function with respect to both variables $x$ and $y$, Lebesgue integrable in $(-\pi, \pi) \times(-\pi, \pi)$ and belonging to the class Lip $\left(\left(\xi_{1}, \xi_{2}\right) ; r\right)$, then the degree of approximation of $f(x, y)$ by $(C, \gamma, \delta)$ means of double Fourier series (1), satisfies

$$
\left\|\sigma_{m, n}^{\gamma, \delta}-f\right\|_{r}=O\left(\frac{1}{(m+1)} \int_{\frac{1}{m+1}}^{\pi} \frac{\xi_{1}(u)}{u^{2}} d u+\frac{1}{(n+1)} \int_{\frac{1}{n+1}}^{\pi} \frac{\xi_{2}(v)}{v^{2}} d v\right)
$$

for $m, n=0,1,2, \ldots$

\section{Conclusion}

We established the degree of approximation of a function $f(x, y)$ belonging to the generalized Lipschitz class by double Hausdorff matrix summability means of its double Fourier 
series in the form of equation (2). If $\xi_{1}=u^{\alpha}$ and $\xi_{2}=v^{\beta}$, then Theorem 1 reduces to Corollary 1 , and as $r \rightarrow \infty$, Corollary 1 reduces to Corollary 2. Independent proofs of Corollaries 1-4 can be developed along the same lines as that of Theorem 1 . Results similar to Corollaries 3 and 4 can be derived for $(E, 1,1)$ means and $(C, 1,1)$ means of its double Fourier series. In this way, we can obtain some more different results by changing $\xi_{1}, \xi_{2}$, and $\mu_{m, n}$ under given conditions. For functions $f(x, y)$ belonging to the Zygmund classes $Z y g(\alpha, \beta)$ and $Z y g(\alpha, \beta ; p)$ discussed in [9], the degree of approximation using double Hausdorff matrix summability means and hence almost Euler means of its double Fourier series can be obtained similarly to Theorem 1 .

\author{
Acknowledgements \\ Not applicable. \\ Funding \\ Not applicable. \\ Availability of data and materials \\ Not applicable. \\ Competing interests \\ The authors declare that they have no competing interests.
}

Authors' contributions

The authors contributed equally and significantly in writing this paper. All authors read and approved the final manuscript.

\title{
Author details
}

'Department of Mathematics, Netarhat Vidyalaya, Netarhat, Jharkhand, India. ${ }^{2}$ Department of Mathematics, Indira Gandhi National Tribal University, Amarkantak, Madhya Pradesh, India. ${ }^{3}$ Department of Medical Research, China Medical University Hospital, China Medical University (Taiwan), Taichung, Taiwan. ${ }^{4}$ Department of Mathematics, Aligarh Muslim University, Aligarh, India.

\section{Publisher's Note}

Springer Nature remains neutral with regard to jurisdictional claims in published maps and institutional affiliations.

Received: 13 August 2020 Accepted: 17 November 2020 Published online: 02 December 2020

\section{References}

1. Acar, T., Mohiuddine, S.A.: Statistical $(C, 1)(E, 1)$ summability and Korovkin's theorem. Filomat 30(2), 387-393 (2016)

2. Chow, Y.S.: On the Cesàro summability of double Fourier series. Tohoku Math. J. 5, 277-283 (1935)

3. Gergen, J.J.: Convergence criteria for double Fourier series. Transl. Am. Math. Soc. 35(1), 29-63 (1933)

4. Hasegawa, Y.: On summabilities of double Fourier series. Kodai Math. Semin. Rep. 15, 226-238 (1963)

5. Khan, H.H., Ram, G.: On the degree of approximation by Gauss Weierstrass integrals. Int. J. Math. Math. Sci. 23(9), 645-649 (2000)

6. Łenski, W., Topolewska, M.: On the rate of strong summability of double Fourier series. Math. Bohem. 123(4), 337-363 (1998)

7. Mohiuddine, S.A., Acar, T.: Advances in Summability and Approximation Theory. Springer, Berlin (2018)

8. Móricz, F., Shi, X:A Approximation to continuous functions by Cesàro means of double Fourier series and conjugate series. J. Approx. Theory 49, 346-377 (1987)

9. Rathore, A., Singh, U.: Approximation of certain bivariate functions by almost Euler means of double Fourier series. J. Inequal. Appl. 2018, 89 (2018)

10. Savaş, E., Rhoades, B.E.: Every conservative double Hausdorff matrix is a $k$ th absolutely summable operator. Anal. Math. 35, 249-256 (2009)

11. Sharma, P.L.: On the harmonic summability of double Fourier series. Proc. Am. Math. Soc. 91, 979-986 (1958)

12. Siddiqui, A.H., Mohammadzadeh, M.: Approximation by Cesàro and B means of double Fourier series. Math. Jpn. 21(4), 343-349 (1976)

13. Stepanets, A.l.: The approximation of certain classes of differentiable periodic functions of two variables by Fourier sums. Ukr. Math. J. 25(5), 599-609 (1973)

14. Stepanets, A.I.: Approximation of certain classes of periodic functions of two variables by linear methods of summation of their Fourier series. Ukr. Math. J. 26(2), 205-215 (1974)

15. Ustina, F.: The Hausdorff means for double sequences. Can. Math. Bull. 10(3), 347-352 (1967)

16. Ustina, F.: The Hausdorff means of double Fourier series and the principle of localization. Pac. J. Math. 37(1), 238-251 (1971)

17. Zygmund, A.: Trigonometric Series, 2nd rev. ed., I. Cambridge University Press, Cambridge (1968) 